\title{
Capacidades para la integración regional en Europa y América Latina: un marco conceptual para el análisis comparativo
}

Edward Best ${ }^{*}$

\section{Introducción}

El presente artículo tiene como objetivo ayudar a elaborar un marco conceptual comparativo para reflexionar acerca de las capacidades necesarias para la gestión de los acuerdos regionales de integración, teniendo en cuenta tanto la estructura común adoptada para un proyecto específico de integración regional, como las consecuencias que esos acuerdos puedan tener para las administraciones centrales de los Estados participantes. Aunque el análisis realizado se deriva principalmente de la consideración de las experiencias europeas y latinoamericanas, se espera que las ideas presentadas tengan una relevancia general'.

Sin embargo, las dificultades metodológicas y conceptuales son numerosas. No existen teorías generalmente aceptadas que permitan contar con una fórmula preconcebida en ninguna circunstancia. La mera formulación de una hipótesis resulta problemática ante la falta de un entendimiento respecto a la naturaleza y a los objetivos de la integración regional en sí. Tanto la variedad como el número de experiencias regionalistas son impactantes. El verano del 95 solamente fue testigo de un nuevo estallido de compromisos debatidos en prácticamente todos los continentes. En mayo, la Asociación del Sudeste Asiático para la Cooperación Regional (SAARC) acordó la posibilidad de una zona de libre comercio. En junio, los Ministros de Comercio de Estados Unidos, Canadá y de 32 países de América Latina y el Caribe pusieron en marcha un programa de trabajo para comenzar a materializar el acuerdo alcanzado en Miami en diciembre de 1994, de crear una Zona de Libre Comercio de las Américas para el 2005. En agosto se estableció la Asociación de Estados Caribenos; los cuatro países del "Visegrad" se comprometieron a liberalizar el comercio de la mayoría de los bienes industriales para 1997 dentro de la Zona de Europa Central de Libre Comercio (CEFTA); y en la Comunidad para el Desarrollo de Africa del Sur (SADC) -originariamente un foro de cooperación frente al apartheid- se comenzó a hablar de la creación de un mercado común para el 2000. En septiembre, los ministros de economía de la (esencialmente política) Asociación de las Naciones del Sudeste Asiático (ASEAN) debatieron la posibilidad de acelerar y extender el programa para crear una Zona Asiática de Libre Comercio (AFTA). Y los Estados miembros del principal foro latinoamericano para la concertación política, el Grupo de Río, que actualmente participan en varias zonas de libre comercio y uniones aduaneras subregionales, en numerosos sistemas de cooperación y en diversos convenios bilaterales, confirmaron su interés en que para el año 2005 se extienda el libre comercio a todo el hemisferio occidental.

Mientras tanto, los miembros de la Unión Europea (UE) estaban también reflexionando sobre lo que harían a continuación. Tenían que hacerlo, ya que para 1996 está programado el comienzo de la Conferencia Intergubernamental cnn el fin de revisar algunas de las disposiciones fundamentales del Tratado de la Unión Europea firmado en Maastricht en febrero de 1992, además de prepararse ante otros desafíos pendientes. Es del caso notar, sin embargo, que por primera vez desde la creación de la primera Comunidad Europea en 1951, esta Conferencia se preocupa más por hacer funcionar las cosas que por encontrar cosas nuevas para hacer. Si bien, la atención pública de la UE se ha visto dominada en los últimos años por los nuevos compromisos establecidos en el Tratado de Maastricht, es cierto que desde comienzos de la década de los noventa la preocupación creciente ha apuntado tanto a los desafíos prácticos, como a los desafíos políticos que enfrenta la integración europea. El fun- 
cionamiento en sí del mercado interno está resultando mucho más difícil de lo esperado, sin contar que la perspectiva de ingreso de nuevos miembros está exacerbando aún más los problemas y las divergencias en materia de eficacia y legitimidad del sistema de gobierno de la UE.

Esas preocupaciones no se limitan a Europa. Puede considerarse un hecho saludable que los problemas de la gerencia de la integración, los que aquí se ha dado en llamar la cuestión de las "capacidades", estén ocupando cada vez más los primeros puestos dentro del temario de la integración de varias regiones del mundo. Por ello, la comparación entre diversas experiencias es probablemente más apropiada y fructífera. Al mismo tiempo, sin embargo, las nuevas circunstancias internacionales que rodean a los proyectos de integración regional pueden, en ocasiones, oscurecer este hecho.

En primer lugar, sigue siendo válido aquello de que todas las experiencias regionalistas son necesariamente singulares. Las políticas de integración -aunque la terminología parezca universal- solamente tienen significado en relación con una realidad regional en particular. No se trata solamente de un problema de nivel de desarrollo, ni de circunstancia política. Como señala Andrew HuRREll (1995), hay quizás cinco dimensiones diferentes, no mutuamente excluyentes del "regionalismo": la "regionalización" ("el crecimiento de la integración social dentro de una región y... el proceso muchas veces no dirigido de la interacción social y económica"); "la conciencia y la identidad regional"; "la cooperación interestatal regional"; "la integración regional promovida por los Estados"; y, como un resultado posible de una combinación de las anteriores, "la cohesión regional". Estas se encuentran presentes en diferentes proporciones en cada experiencia regionalista, las cuales están, a su vez, insertas en un conjunto mucho más amplio de interacciones sociales y económicas, de relaciones culturales e históricas que en forma directa y única conforman los motivos, el alcance, las formas institucionales y las capacidades de gestión necesarias para un proyecto de integración. ¿Acaso no se corre todavía el peligro de repetir lo que Rolf LANGHAMmer solía llamar muy atinadamente la "falacia de la transposición" de la experiencia de la Comunidad Europea (o de cualquier otra)?

En segundo lugar, en el nuevo período de la liberalización tanto unilateral como multilateral, y en la época posterior a la Guerra Fría, podría legítimamente preguntarse en qué medida la integración, en el sentido de la Comunidad Europea de construir una unión regional preferencial cada vez más estrecha (y permanente), sigue apareciendo aún en el temario, en otro lugar que no sea Europa (e incluso allí, algunas partes la están cuestionando). ¿No es acaso la globalización la nueva realidad, en la cual la "integración" económica se refiere a un fenómeno conducido por el mercado y no promovido por el Estado y donde los acuerdos preferenciales regionales son considerados, en el mejor de los casos, como etapas transitorias dentro del proceso de establecimiento del libre comercio multilateral?

Sin embargo, el mundo está todavía muy lejos del libre comercio multilateral y de la competencia mundial perfecta. Desde la perspectiva de la política del desarrollo nacional, la integración regional ofrece un conjunto de opciones preferenciales que pueden resultar transitorias, como lo sugiere la nueva ortodoxia, pero que pueden también no serlo. ¡De una u otra forma es evidente que sí se están persiguiendo tales opciones!

Además, si bien es cierto que escs proyectos de carácter preferencial pueden surgir por motivaciones y circunstancias muy diversas, esto no necesariamente significa que no haya lugar para un marco comparativo basado en conceptos generales. Se puede sugerir, asimismo, que objetivos políticos conceptualmente diferentes, tales como el de "unión aduanera" o el de "libre circulación de las personas" pueden tener diferentes significados, impactos y requerimientos, dependiendo de la realidad regional en la que tengan lugar. Para citar un caso extremo, el objetivo de la libre circulación de personas, o un mercado laboral común, ha tenido resultados sumamente diferentes en dos sistemas (sub)regionales de Europa. Mientras que en la década de los 50 los países nórdicos lo lograron sin dificultad alguna y sin ni siquiera tener instituciones supranacionales, resultó virtualmente imposible dentro de la Comunidad/Unión Europea, donde la eliminación de los controles sobre las personas sigue siendo muy problemática -aun entre los signatarios de los acuerdos Schengen.

Prosiguiendo con esta lógica, se propone:

- comparar los proyectos de integración regional según su nivel de ambición;

- establecer para cada tipo de proyecto de integración, cuáles son sus requerimientos generales en términos de capacidades de gestión pública en el ámbito común y nacional, e

- indicar las formas de cómo las necesidades y capacidades de gobierno de los distintos grupos de países comprometidos en esos proyectos pueden relativizarse en función de sus circunstancias e intereses.

\section{Proyectos de Integración Regional y Requerimientos de Política}

\subsection{Niveles de ambición integradora}

La idea de "ambición" puede interpretarse de diversas maneras con respecto a la integración económica. Cuando se identifica la integración con el libre comercio, se está haciendo refe- 
rencia primordialmente al alcance de la liberalización. En las Américas de la actualidad parece absolutamente natural que los esquemas preferenciales regionales aparezcan como un nivel de opción, o como una etapa en el camino hacia la liberalización hemisférica y, en última instancia, mundial. La Comisión Económica para América Latina y el Caribe de las Naciones Unidas (CEPAL), en su documento de 1994 sobre el "regionalismo abierto" sostiene que: "Cuando exista un mayor número de países bien encaminados por la senda de la estabilización habrá mayores posibilidades de llegar a acuerdos más amplios, es decir, de multilateralizar el proceso. En este sentido, aun cuando se reconoce la heterogeneidad que caracteriza los compromisos integradores subregionales en la actualidad, no es utópico plantear la posibilidad de impulsar un proceso de integración que culmine -sin fecha preestablecida- en la constitución de una zona de libre comercio de alcance regional, y acaso hemisférico" (CEPAL, 1994: 14).

Sin embargo, las autoridades nacionales pueden también elegir, en cierta medida, entre niveles analíticamente distinguibles de ambición en cuanto a la intensidad deseada del acuerdo preferencial regional, además del alcance de los miembros que lo integrarán.

En este sentido, los conceptos o etapas clásicas de la integración -libre comercio, unión aduanera, mercado común, unión económica- siguen siendo válidos en gran medida. Se puede ir más allá, como en la taxonomía elaborada por Jacques Pelkmans (1993), que puede ser de utilidad para definir en mayor detalle, o bien los límites que se intenta poner, o bien los requerimientos políticos de ciertos acuerdos determinados (incluyendo el alcance de la cobertura). Un primer nivel de preferencialismo se desglosa en preferencialismo sectorial parcial, integración sectorial, sistemas preferenciales no recíprocos, preferencialismo parcial recíproco. Las áreas de libre comercio se dividen en aquellas que abarcan toda la industria y las que abarcan todos los sectores. Una unión aduanera se diferencia en varias etapas de integración del mercado de productos. Más allá de esto, se tiene también la integración del mercado de los servicios, del capital y del trabajo para alcanzar un mercado común completo y varias etapas y opciones respecto de las transiciones hacia la Unión Económica y la Unión Monetaria ${ }^{2}$.

Debe señalarse, sin embargo, que algunos aspectos de un mercado común pueden existir en ausencia de otros. En los países nórdicos, por ejemplo, se llegó a altos grados de integración social, independientemente de la integración del mercado de bienes (que solamente se alcanzó para los bienes industriales y esto únicamente dentro de la EFTA): lo más notorio fue la creación de una Unión de Pasaportes, que implica la circulación totalmente libre de las personas junto con un mercado común laboral que abarca la armonización de la seguridad social. La cooperación monetaria tampoco depende necesariamente de la integración del mercado.

la cooperación política intergubernamental puede también proceder en forma bastante independiente de toda integración económica. En el caso de la ASEAN se alcanzó una cooperación política significativa y una concertación de las posiciones negociadoras frente a terceros países que, durante mucho tiempo, estuvieron acompañadas de una falta de interés en la integración económica regional. Parece poco probable, sin embargo, que se pueda contemplar la integración política, en el sentido de una fusión progresiva de las autoridades públicas, como algo ajeno a una unión económica virtualmente completa.

\subsection{Requerimientos de politica}

La taxonomía de Pelkmans es, a grandes rasgos, progresiva, porque cada nivel implica un nivel más alto de ambición y mayores demandas en cuanto a medidas positivas de integración. Es también acumulativa porque cada una de las distintas etapas constituye en sí, como regla general, un logro previo indispensable.

Es muy tentadora la simplificación que hace suponer que las formas de integración con niveles "más bajos" de ambición (llegando hasta el área de libre comercio completa) solamente requerirán una "integración negativa", es decir, medidas para eliminar las barreras, mientras que las formas restantes requerirán una "integración positiva", ya que todas ellas necesitan irremediablemente la adopción positiva de normas y políticas comunes, comenzando con un arancel externo común. En realidad, sin embargo, hasta la forma "más baja" de preferencialismo parcial necesariamente crea algunos requerimientos positivos, tales como las normas de origen comunes. Los acuerdos de libre comercio desarrollados están acompañados frecuentemente por acuerdos muy minuciosos en materia de reglamentaciones técnicas, certificaciones, prácticas comerciales, solución de controversias, etcétera. Por supuesto que algunas medidas recién entran en juego a partir del nivel de unión aduanera hacia "arriba", siendo el caso más evidente el arancel externo común, pero el punto más importante es que hay una diferencia cualitativa en el proceso.

Se debe tener en cuenta, en particular, el grado de dinamismo, ya que afecta tanto al alcance como a la intensidad del acuerdo. Los acuerdos de liberalización simple son básicamente estáticos. De la misma forma que no haya probablemente un deseo político, por parte de los Estados participantes, de aceptar la pérdida abierta del control nacional que significa un arancel común o más, no hay inserta en ese acuerdo una dinámica para el cambio (lo cual no significa que las autoridades nacionales no vayan a tener que enfrentarse a los problemas del cambio, a medida que el libre comercio aporte cambios estructurales a las economías domésticas). Los proyectos más ambiciosos son, por otra parte, inevitablemente dinámicos. Los tratados 
constitutivos de esos acuerdos se caracterizan, justamente, por tener objetivos generales que deben alcanzarse progresivamente mediante la generación de nuevas normas. Aunque esto no fuera así, el establecimiento de una unión aduanera es prácticamente seguro que desencadene un proceso de "derrame" para el que habrá que convenir nuevas medidas para enfrentar los obstáculos no arancelarios y las distorsiones de la competencia, o para responder a los graves desequilibrios económicos y sociales exacerbados o causados incluso, por el propio proceso de integración. En algunas áreas, las vinculaciones políticas resultan evidentes. Si los países acuerdan liberalizar la circulación de las personas, por ejemplo, entonces las consecuencias alcanzarán inevitablemente la cobertura de los controles fronterizos externos, las políticas comunes frente a personas de terceros países, la cooperación aduanera, la cooperación policial y otras.

\section{Adecuación de las capacidades a las necesidades}

\subsection{Necesidades de gobernación}

Existen varios trabajos que analizan los procesos de integración en términos generales de los requerimientos del sistema, por ejemplo, la aplicación por CAPORASO (1974) del trabajo de ALMOND y Powell sobre sistemas políticos: las funciones de conversión, las capacidades y el mantenimiento y adaptación de los sistemas; o la división de JACOBSON (1984) de las funciones de las organizaciones internacionales en "cinco categorías principales: informativa, normativa, creadora de normas, supervisora de normas y operativa".

Desde una perspectiva similar, se pueden identificar una serie de requerimientos funcionales más específicos que deben ser satisfechos por las estructuras y procesos que se desarrollen en todo acuerdo regional ${ }^{3}$. Estos incluyen (BEST, 1994):

Toma de decisiones:

- Normas para la enmienda de los acuerdos constitutivos.

- Decisiones para la formulación de políticas (normativas).

- Toma de decisiones para la aplicación de las normas.

Ejecución:

- Ejecución práctica.

- Monitoreo.

- Solución de controversias.

- Obligación de cumplimiento.

\section{Mantenimiento del "consenso" y la legitimidad:}

- Aspectos de procedimiento/políticos: transparencia, información al público, participación de sectores, parlamentos, regiones, etc.

- Sustantivo: evitando los desequilibrios excesivos entre costos y beneficios.

\section{Desarrollo de politicas:}

- Retroalimentación y evaluación.

- Planificación futura y previsión de problemas.

Aprendizaje y adaptación de la organización:

En el ámbito de las administraciones centrales de los Estados Miembros, las necesidades principales corresponden, en gran medida, a lo precedente:

- Acceso a la información: (inteligencia).

- Concertación de posiciones nacionales (preparación para las negociaciones).

- Participación efectiva en negociaciones multilaterales (influencia sobre la toma de decisiones).

- Control sobre la ejecución y divulgación de la información (ejecución).

- Asegurarse el apoyo público (legitimidad).

Estas necesidades quedarán definidas no solamente por el nivel de ambición integradora en términos abstractos sino también por las circunstancias específicas de los países involucrados.

La interrogante básica debe replantearse, entonces, de la siguiente manera, ¿cuáles son las capacidades de gestión pública que se requieren para la ejecución del conjunto de objetivos $\mathrm{X}$ de integración, entre el conjunto de países y en los ámbitos comunitario, nacional y ministerial? Lo importante es el grado de dificultad planteado para un grupo de países en particular, y para cada administración participante, de modo de enfrentar en forma eficaz un conjunto determinado de objetivos de integración en un momento dado. La variable tiempo es decisiva. La integración de los mercados es más fácil en tiempos de auge económico. En los períodos de bajo grado de nacionalismo por parte de los actores dominantes, el grado de intensidad del método integrador será más alto. Esto resulta evidentemente clave para entender las primeras etapas de la integración europea.

Las variables que conforman la complejidad de la ejecución de los objetivos de la integración y que deberían influir sobre el desarrollo de las capacidades para satisfacer las necesidades de gobierno, pueden identificarse provisoriamente de la siguiente manera ${ }^{4}$. Debe señalarse que esto no implica relaciones simples o directas. El efecto real depende de la combinación específica de las variables en cuestión. 


\section{El "número" de Estados miembros}

Se supone, con frecuencia, que las perspectivas de cooperación disminuyen siempre a medida que el número de participantes aumenta. En efecto, cuanto mayor es el número, cabe esperar que la toma de decisiones se vuelva más difícil en aquellas oportunidades que requieren unanimidad o en áreas de sensibilidad extrema. Sin embargo, la relación no es simple. Sobre todo en condiciones de votación por mayoría, un número mayor de participantes puede facilitar la toma de decisiones, en la medida en que ofrece mayores oportunidades para intercambios y compensaciones (side-payments), al igual que para la formación de coaliciones (MILNER, 1992). En todo caso, el impacto real va a depender también de otros factores relativos a las características de los países en cuestión.

Esta cuestión está en el centro del debate europeo actual. En América Latina, por otra parte, el tema del número de miembros no parece haber surgido como tal. Los proyectos de mayor nivel de ambición -Mercado Común Centroamericano (MCCA), Grupo Andino, Mercado Común del Sur (MERCOSUR)- no han excedido hasta ahora de cinco miembros, mientras que la organización más amplia -el Sistema Económico Latinoamericano (SELA), que incluye a toda América Latina y al Caribe- carece de funciones normativas.

\section{Los "tamaños relativos" de los países}

La distribución del poder en este respecto puede tener efectos de diferente índole.

La presencia de un país mucho más grande que los otros, por ejemplo, puede producir un liderazgo bien acogido o una dominación temida, según las circunstancias. Quizás el ejemplo más claro sea la actitud cambiante y compleja en América Latina hacia Estados Unidos, relativa a la cooperación hemisférica.

Cuando se percibe una división entre países pequeños y uno o más países de mayor tamaño, los primeros siempre tendrán interés en asegurar que todos los miembros cumplan con los compromisos y que se tomen debidamente en cuenta los intereses de todos. Se puede escoger entre por lo menos dos alternativas, según la naturaleza básica del proyecto común. Por un lado, como en el caso de la Comunidad Europea, se puede adoptar un sistema jurídico-institucional con fuertes poderes supranacionales, en el que los países pequeños aceptan la votación por mayoría cualificada, sabiendo que la Comisión asegura la participación de todos en la formulación de políticas, mediante su derecho exclusivo de iniciativa legislativa $y$, junto con el Tribunal, vela por el cumplimiento. Por el otro lado, cuando no exista un sistema parecido, no queda más que el consenso. En el sistema intergubernamental adoptado en el MERCOSUR, por ejemplo, el consenso ha sido explícitamente visto en el país miembro más pequeño (Uruguay) como "el mecanismo que ha dado la garantía por igual a todos los Esta- dos Partes de que ninguna decisión será tomada sin su aceptación, expresa o tácita" (PÉREZ OTERMín, 1995: 91).

\section{Los "niveles relativos de desarrollo"}

La existencia de diferencias significativas en el nivel de desarrollo exacerbará los problemas de distribución que abundan en los proyectos de integración.

Tal fue notoriamente el caso en la primera ola de proyectos de integración en América Latina. La percepción de una distribución desequilibrada de costes y beneficios contribuyó en forma significativa a la crisis del MCCA (incluyendo una breve guerra entre El Salvador y Honduras en 1969), a la decadencia de la Asociación Latinoamericana de Libre Comercio (ALALC) y, en gran medida, como una respuesta a esta situación, a la constitución del Grupo Andino en 1969.

\section{"Alcance" de la cobertura}

La inclusión de aquellas áreas que los participantes consideran sensibles puede dificultar el acuerdo en algunos casos, aunque también es cierto que cuanto más amplio es el alcance, mayores son las oportunidades de negociación.

\section{"Impacto"s}

No solamente se trata de la fuerza del cambio que se produce en las economías. La complejidad deriva también del tipo de impacto: en particular, si los costos están ampliamente compartidos o concentrados en grupos determinados (y que éstos estén más o menos organizados, etc.). Por otra parte, hay que tener en cuenta la incertidumbre del impacto de la integración -y el tiempo que transcurre antes de que puedan manifestarse los beneficios tangibles.

\section{El grado de "interdependencia" real}

Es bien conocida también la debilidad que implica el bajo nivel de intercambios económicos pre-existente en la mayoría de los proyectos de integración regional que han sido iniciados entre países en vías en desarrollo.

El caso más notorio en las Américas ha sido quizás el Grupo Andino. En un ejercicio reciente en el Banco Mundial se ha aplicado el índice de complementariedad comercial de MichaELY para medir la probabilidad de que un acuerdo regional determinado, en el momento de su establecimiento, tenga éxito en fomentar el comercio entre las partes. Para la Comunidad Económica Europea el valor es 0,53 . La cifra para el Grupo Andino es 0,07. MERCOSUR se sitúa en medio, con 0,29, mientras que NAFTA supera la cifra europea, con un 0,56 (World Bank, 1995: 20-21). De hecho, de un porcentaje de comercio intrarregional de un 1,8 por 100 en 1970 , apenas se subió a 4 por 100 en 1980 , y a 5 por 100 en 1990.

En la nueva fase de integración, este porcentaje ha llegado a 10 por 100 en 1994 , debido principalmente al fuerte aumento de las relaciones económicas entre Colombia y Venezuela. Este 
nuevo dinamismo ha caracterizado el comercio intra-regional en toda América Latina. Entre los factores que pueden haber influido en este dinamismo se puede senalar tanto la revitalización de esquemas de integración existentes y la creación de nuevos proyectos, como el hecho de que el proceso generalizado de liberalización de los 80 haya revelado áreas geográficas de comercio "naturales" que anteriormente fueron encubiertas por la protección doméstica de industrias de sustitución de importaciones (CEPAL, 1994b; IDB, 1995: 3).

Un alto grado de interdependencia económica, sin embargo, no necesariamente obliga a formas de integración más intensas, como demuestra el caso nórdico y la posición actual de Suiza y Noruega.

En todo caso, no se trata meramente de un asunto de interdependencia económica. Es de igual trascendencia la medida en que el sistema se base en niveles significativos de interacción transgubernamental o transnacional (en el sentido de "comunicación social" de Karl DeUTSCH) y la importancia de los vínculos políticos e incluso de las alianzas militares (ver por ejemplo: MANSFIELD, 1993).

\section{El "marco politico"}

¿Existen metas políticas -u obstáculos políticos- que influyen en el proceso de integración? ¿Existe una amenaza externa común, o consciencia de impotencia internacional propia, que sea un aliciente para unirse?

El papel de Estados Unidos en el hemisferio occidental ha contribuido en muchas ocasiones al fortalecimiento de la idea de la integración latinoamericana, a veces con una cierta dimensión política. Fue con este espíritu con el que se creó en 1976, en pleno auge del "tercermundismo", el SELA incluyendo a Cuba y excluyendo a Estados Unidos.

Sin embargo, la agenda inter-americana siempre ha incluido también aspectos positivos de cooperación (incluyendo en muchos casos una alianza anticomunista), mientras que la integración ha sido minada por la existencia de diferencias políticas y rivalidades nacionales, incluyendo problemas territoriales entre los propios países.

Pasada la ola de liberalización y democratización en casi toda la región, parece poco probable que haya un resurgimiento de un integracionismo con ánimo de oposición a los Estados Unidos, por lo menos en los terrenos económico y político. Lo que sí se puede esperar es que haya una renovada atención a la dimensión cultural -y de identidad- de la integración. Es posible que la cuestión de Cuba sea relevante otra vez en este respecto.

De hecho, tanto en las Américas como en las Europas, la desaparición de una amenaza externa nos está obligando a repensar el integracionismo, y plantear más abiertamente preguntas a veces muy delicadas sobre las bases reales de los esquemas regionalistas.

\section{"Percepciones y valores"}

¿En qué medida son similares las percepciones dominantes en cada Estado en cuanto a la naturaleza, los objetivos y la importancia del propio proceso de integración?

¿En qué medida difieren las tradiciones constitucionales, los sistemas políticos, las políticas económicas?

¿Se perciben más las identidades comunes o las diferencias entre los países? ¿Existen más similitudes o contrastes en la estructura social y en los sistemas de valores nacionales?

Muchas de las variables sugeridas anteriormente se aplican también en el ámbito nacional (y subnacional). No obstante lo cual se podrian agregar:

- La medida de las interdependencias que actúan en el ámbito nacional:

- El número de actores afectados.

- El alcance de los vínculos políticos.

- La sensibilidad de los principales sectores participantes, la profundidad del cambio esperado y la probable fuerza de la resistencia.

- Las actitudes nacionales hacia la integración (los sentimientos, ¿son positivos o negativos, están unidos o divididos?).

\subsection{Capacidades de gobierno}

Estas necesidades se deben satisfacer mediante un "manejo" adecuado de las capacidades.

A todo los niveles está presente una dimensión cuantitativa básica ('hay suficientes personas cualificadas, que cuenten con el apoyo material suficiente para enfrentar lo que está sucediendo?), asi como los aspectos técnicos más cualitativos (ison suficientes los sistemas de comunicación?). Igual importancia reviste, no obstante, la estructura y la organización del sistema de integración.

En el ámbito común es relativamente fácil establecer las taxonomías y las matrices referidas a la naturaleza y las facultades de las instituciones regionales y a sus formas de interactuar con las autoridades nacionales (y subnacionales), las asambleas y el público (BEST, 1994). Resulta mucho más difícil formular enunciados concretos acerca de cómo éstos se deberían ajustar a las necesidades, incluso en un nivel teórico general. Este tema ha sido poco elaborado, especialmente desde la perspectiva abiertamente "funcional" adoptada aquí.

Pelkmans (1986) identifica sugestivamente "siete propiedades institucionales esperadas de la integración de las economías mixtas":

- Los compromisos estrictos con respecto a la transferencia de ciertas funciones económicas a los agentes públicos centrales y las restricciones de algunos instrumentos nacionales. 
- Una presencia ínfima de salidas y de cláusulas de salvaguardia y de escape.

- "La supervisión común y obligatoria de las cláusulas de escape y de salvaguardia y, posiblemente, incluso una autorización central previa".

- Ninguna posibilidad de "sacarse de encima" las funciones "medulares" transferidas, si bien hay mayor indulgencia en cuanto a las acciones cooperativas complementarias.

- Duración ilimitada de los compromisos.

- Una prescripción precisa de los procedimientos y los pasos en el período de transición.

- "Modalidades algo sofisticadas de revisión legal".

MOLLE, por su parte, nos propone la siguiente "jerarquía de cooperación" en las políticas: información, consulta, coordinación (adaptación o armonización de las leyes nacionales y las normas administrativas, acuerdos que tengan en cuenta objetivos variables, etc.) y unificación (ya sea mediante el reemplazo de los instrumentos nacionales por instrumentos de la unión, o mediante la adopción de instrumentos idénticos para todos los socios). Sugiere que hay una cierta relación entre los estados de integración y esta jerarquía de manera tal que "las formas más altas de integración requieren instrumentos más obligatorios". Advierte, no obstante, que "ni la mezcla óptima entre las medidas de la unión y las nacionales, ni las áreas en que una integración ulterior será más beneficiosa pueden determinarse de antemano sobre una base teórica" (Molle, 1994: 20-23).

\subsection{Las dimensiones común y nacional}

La "dimensión nacional" es aún más difícil de encarar dentro de un marco comparativo. Por una parte, está conformada por una variedad aún más amplia de circunstancias. Por otra, puede parecer todavía más difícil el separar las "necesidades de integración" de las otras modalidades de capacity-building: ¿en qué difieren los requerimientos específicos para la "integración" de las capacidades que se requieren para enfrentar cualquier otro tipo de relación internacional?

Resulta evidente que, en primer lugar, las implicaciones reales para las administraciones nacionales estarán en función de las características precisas del sistema global adoptado por los Estados participantes. Más importante aún, la naturaleza de la relación entre las administraciones del ámbito común y las del ámbito nacional, variará por sí misma de acuerdo con el nivel de ambición específico y el grado de interdependencia real.

Primero, como regla general, "las políticas comunes requieren instituciones comunes más fuertes cuanto mayores sean las complicaciones de las diferentes etapas políticas: preparación, formulación y ejecución" (Molle, 1994: 15). Debe señalarse una vez más, sin embargo, que no hay relaciones simples y no existe ninguna necesidad de una relación jerárquica vertical simple, fuere cual fuere la entidad de las facultades de las instituciones comunes en sus respectivos campos de competencia. El caso europeo sugiere precisamente que una integración más profunda no significa necesariamente que el papel práctico de las administraciones nacionales sea menos importante, sin importar cuáles son los nuevos niveles de identificación social e incluso los cambios en lealtades políticas que puedan tener lugar. Por el contrario, las capacidades nacionales se han vuelto cada vez más vitales para el éxito del proyecto en su totalidad.

El programa estratégico de Europa para "hacer que funcione el mercado único", a partir del Informe Sutherland de 1992, es explicíto en el nuevo énfasis puesto en un "partenariado" de las relaciones tanto entre la Comisión y las administraciones nacionales, como entre éstas. Vale la pena recordar, asimismo, que la Comunidad Europea, en general, no es tan jerárquicamente vertical como a menudo se la considera. Las instituciones centrales pueden verse, por el contrario, como el centro de una red borizontal (como la maza de una rueda), un "federalismo político invertido sin federalismo administrativo": primero, contrariamente al sistema alemán, la institución de la $\mathrm{CE}$ que comprende representaciones de los Estados miembros (el Consejo) tiene mayor peso que la institución formada por individuos directamente elegidos por los ciudadanos (el Parlamento) y, segundo, son principalmente las administraciones nacionales y subnacionales las que ejecutan las políticas comunitarias (ZILLER, 1993: 482-3). Incluso el sistema jurídico, quizás el aspecto más federal de la Comunidad Europea, no es indisolublemente jerárquico. La doctrina de la primacía del Derecho Comunitario, en realidad, no ha sido aceptada sin cuestionamientos aun en los países que forman el eje central de la integración europea. La declaración por parte del Tribunal Constitucional Federal de Alemania, que en el contexto de su decisión de 1993 establece la constitucionalidad del Tratado de Maastricht para Alemania y la "relación de cooperación" entre el propio Tribunal y el Tribunal de Justicia de Luxemburgo es una "negación lisa y llana (y renovada) de la supremacía absoluta del Derecho Comunitario y de su órgano judicial supremo" (HeRDEGen, 1994: 239).

Segundo, muchas veces se pasa por alto la importancia y las implicaciones de la "dimensión inter-nacional". En un mercado común pleno, la protección de los intereses "propios" de un país depende también de la eficacia de las otras administraciones nacionales en esferas clave, tales como el control y la certificación de los productos, o los controles aduaneros y de inmigración en las fronteras externas. No se trata solamente de un asunto de mutuo reconocimiento sino, to que es crucial, de confianza mutua.

Tercero, cuanto mayor es la intensidad de la relación de integración, "más grises" tenderán a ser las líneas divisorias analíticas y prácticas entre la formulación de decisiones comunes y nacionales. No es solamente una cuestión de autonomía. Cuan- 
to más profundo y más extenso es el proceso de integración, más importante será para las administraciones poder desarrollar nuevas capacidades para asegurar la eficacia no sólo de la ejecución nacional, sino también de la participación en la toma de decisiones y en la planificación política; como lo señala ZILLR: "La adaptación en Europa consiste en reforzar la capacidad de anticipación en el seno de la Unión Europea" (ZILLR, 1994: 242; énfasis del autor). Al mismo tiempo, la relación entre los ámbitos común y nacional, en el contexto de un proceso avanzado de integración, es de una separación decreciente.

Gran parte de la atención se ha centrado, tradicionalmente, en las capacidades de integración en el ámbito común y sólo cuando éstas se negocian entre gobiernos. En otras palabras, las teorías se relacionan primariamente a las decisiones "constitucionales" entre los países integrantes y son, por naturaleza, intergubernamentales y, consecuentemente, similares a otras negociaciones internacionales. Sin embargo, los mismos conceptos no se aplican necesariamente a los niveles de toma de decisiones para la elaboración de políticas y su ejecución que comprenden patrones de interacción completamente diferentes en el contexto de un proceso de integración regional comparado con un marco meramente cooperativo.

La diferenciación entre modelos de negociación es clave. El modelo de Putnam (1988) que dice que la diplomacia es un "juego a dos niveles", en el que un negociador único se enfrenta primero con sus interlocutores a nivel doméstico y luego con sus contrapartes internacionales, puede ser válido respecto del proceso de negociación de tratados de prácticamente cualquier clase. Sin embargo, este modelo es demasiado simplista en la mayoría de los casos dentro de la $\mathrm{CE}$, donde los "amplios intercambios que tienen lugar entre los ámbitos nacional e internacional ya no son más canalizados a través de un único negociador nacional sentado frente a ambos tableros de juego como lo supone el modelo de PUTNAM. En realidad, este negociador ejerce bastante poco control sobre muchas de las interacciones que están teniendo lugar a distintos niveles. En pocas palabras, debe recurrirse a otros modelos cuando los juegos se vuelven tan complejos que los grupos de interés nacionales, los grupos de interés internacionales y las organizaciones gubernamentales internacionales se unen a los negociadores nacionales en los dos tableros de juego" (ATKINSON y Coleman, 1992: 172).

Resulta esencial la participación activa de las unidades pertinentes de la administración nacional (o de las autoridades subnacionales, por ejemplo) lo antes posible en el curso del proceso tanto para promover los intereses nacionales, como para evitar los problemas previsibles de la ejecución. Este desafío se puede enfrentar con una claridad, confianza y calma. "Para la administración central se trata, por una parte, de disponer de las estructuras competentes y especializadas para cada una de las atribuciones comunitarias y, por otra parte, de permitir una coordinación inter- ministerial eficaz para defender de manera apropiada las posiciones del gobierno francés" (OBERDORFF, 1995: 29). No obstante y especialmente en el caso de los Estados menos centralizados, el desafío de la gestión pública puede verse también desde otra óptica. No solamente "las administraciones nacionales comienzan a utilizar la administración comunitaria para procesar los problemas internos". Se puede esperar que se produzca un efecto "desestabilizador" sobre los sistemas y patrones administrativos nacionales establecidos a medida que los gobiernos en forma individual, las regiones o los gobiernos locales e incluso parte de los mismos traten de lograr, cada vez más, en el ámbito europeo, lo que no fueron capaces de alcanzar dentro del contexto nacional (TOONEN, 1992: 111)6. En dichos contextos, la coordinación de políticas (MetCalfe, 1993b) es un elemento de importancia central', entre las múltiples necesidades que enfrentan las administraciones ante los nuevos retos de la integración.

\section{Conclusiones}

Todos los proyectos de integración regional requieren algún grado de gerenciamiento. De hecho, la primera capacidad requerida para hacer que la integración funcione es aceptar que existe la necesidad de pensar sobre las capacidades, cuyo objetivo es no hacer ni tan poco ni demasiado, sin estar encegado ni por las tentaciones de imitar modelos institucionales de fuera ni por presunciones según las cuales no existe necesidad de ninguna institución pública y de ninguna generación de capacidades en absoluto.

La comparación puede ofrecer un instrumento valioso para lograr este objetivo. Sin embargo, las diferentes circunstancias y objetivos de los acuerdos regionales no pueden ser ignoradas ni tampoco simplemente reconocidas. Este artículo ha, por lo tanto, intentado contribuir a crear un marco conceptual que activamente incorpora las múltiples diferencias involucradas.

El marco toma como punto de partida el nivel de ambición de un proyecto de integración acordado entre Estados en términos de la pretendida intensidad de sus vínculos recíprocos. Y afirma la necesidad de tener en cuenta algunos principios generales que conciernen a las políticas y a los instrumentos requeridos para la estabilidad de las respectivas modalidades de proyecto. Más allá de esto, sin embargo, se enfatiza el hecho de que el logro de un conjunto particular de objetivos de integración será más o menos problemático en función de determinados factores que inciden en el acuerdo regional. Las complejidades resultantes podrán, a su vez, ser tomadas como puntos de referencia para establecer una correspondencia estable entre necesidades de gobernación y capacidades de gobernación.

En ningún caso se está sugiriendo que cuantas más "capacidades", mejor. Si se trata de un alto nivel de ambición integra- 
dora, las administraciones deberían examinar todo lo que comporta esa elección y tomar medidas de manera a adaptar las capacidades en los ámbitos común y nacional. De lo contrario, se corre el riesgo de tener un "déficit" de capacidades, cuyo resultado será la ineficacia y la ineptitud para contrarrestar las presiones desestabilizadoras. Éste ha sido un factor clave que explica la escasa aplicación de los acuerdos de integración entre paises en desarrollo: "la mayoría carecía de los mecanismos institucionales necesarios para alcanzar los objetivos; esto resultaba evidente por las incoherencias existentes entre la legislación nacional y los compromisos de integración, por la ausencia de mecanismos potentes para obligar a cumplirlos y por los procedimientos poco eficaces para la resolución de controversias y la falta de mecanismos de compensación para abordar las preocupaciones en materia de distribución" (DE LA TORRE y Keuly, 1992: 34). Si los países no aspiran, en cambio, a un grado de integración muy intenso (o si no existe un grado mínimo de consenso entre ellos para alcanzarlo), entonces la creación de instituciones nuevas y la asignación de nuevos recursos puede crear un "superávit" de capacidad que resulta costoso y que puede debilitar incluso la legitimidad del acuerdo regional.

En la práctica, la definición y readecuación de los sistemas será siempre el resultado de la negociación política. El análisis de las necesidades y capacidades en los términos anteriormente descritos puede contribuir a tales debates. Lo que más importa, en todo caso, es que la adecuación de capacidades a necesidades se realice en los propios términos de la realidad regional en cuestión.

El "modelo" de la CE, por ejemplo, no ha sido relevante en todos los casos de integración siquiera en Europa. A partir de la década de los setenta, la Asociación Europea de Libre Comercio (EFTA) experimentó una serie de movimientos hacia la CE/UE: por parte del Reino Unido y Dinamarca en 1973, de Portugal en 1986 y de Austria, Finlandia y Suecia en 1995. En la actualidad, la EFTA comprende solamente Islandia, Liechtenstein, Noruega y Suiza. En algunos círculos se considera (bajo la influencia del pensamiento neofuncionalista) que la EFTA "fracasó" porque carecía del tipo apropiado de instituciones (o del liderazgo, etcétera) necesarios para un desarrollo mayor del proceso. Pero los "eftanos" en realidad no querian nada más que lo que firmaron en 1960. La estructura puramente intergubernamental de la EFTA era perfectamente suficiente para el bajo grado de complejidades que se presentaban: la EFTA tenía un bajo grado de ambición integradora; a excepción de uno, los Estados miembros eran prácticamente iguales y presentaban niveles de desarrollo igualmente altos; y sus sistemas internos eran tales que aseguraban que los gobiemos cumplieran con los acuerdos.

Del mismo modo, el tipo de "acción nacional paralela" (Nielsson, 1990), practicado por Dinamarca, Finlandia, Islandia, Noruega y Suecia en la Cooperación Nórdica, sin instituciones supranacionales o sistema jurídico alguno, se adecuaba completamente al tipo de objetivos que se perseguía. El sistema institucional que se creó surgió con el fin de "gestionar colectivamente el transnacionalismon ${ }^{n}$, en forma tal que reflejaba las condiciones específicas y la historia de los países nórdicos (y, en parte, también sus culturas políticas internas) (SundELIUS, 1978; SUNDELIUS y WIKLUND, 1979). En efecto, si se compara el papel marcadamente diferente de las instituciones nórdicas con el de las instituciones de la Comunidad Europea resulta claramente evidente la secuencia en que la cooperación fue creciendo: el proceso comenzó con el transnacionalismo, continuó con un transgubernamentalismo creciente, para finalizar con la creación de las instituciones intergubernamentales. Obsérvese asimismo que la primera institución, el Consejo Nórdico creado en 1952, fue un órgano interparlamentario. El Consejo Nórdico de Ministros se creó en 1971, con el objetivo de consolidar lo que ya se había logrado.

Es dudoso, en cambio, que los Estados miembros de la Comunidad Europea pudieran haber logrado objetivos de tan alto grado de ambición sin un sistema legal e institucional poderoso, al tiempo que esos objetivos ambiciosos eran considerados deseables, hasta inevitables, precisamente debido a la relación entre los países asociados. Actualmente, la Unión está enfrentando un proceso de readecuación de capacidades para responder a una serie de presiones causadas por su dinámica interna y por cambios profundos en su entorno.

En América Latina, también, se están dando procesos de ajuste. En los países miembros del MERCOSUR se han proseguido las discusiones relativas a la suficiencia de la estructura institucional completamente intergubernamental establecida en el Protocolo Adicional al Tratado de Asunción de 1991, la cual fue adoptada en Ouro Preto (Brasil) en diciembre de 1994. En América Central, la Cumbre Presidencial de San Salvador de marzo de 1995 ordenó una revisión de la estructura institucional del "Sistema de Integración Centroamericano". Y en septiembre de 1995, los Presidentes de Bolivia, Colombia, Ecuador, Perú y Venezuela formalizaron la transformación del Pacto Andino en el "Sistema Andino de Integración" y adoptaron una serie de reformas institucionales dirigidas a afrontar los nuevos desafíos planteados por la integración. Sería prematuro, sin embargo, intentar emitir juicio alguno sobre los resultados de estos procesos.

Por último, hay que reconocer, sin embargo, que nunca se sabe. Por cierto, se deben identificar las necesidades "técnicas" de un acuerdo regional lo más claramente posible, teniendo en cuenta las relatividades impuestas por las circunstancias políticas. Pero el desafío va mucho más allá del mero diseño de organizaciones y de la dedicación de recursos para ejecutar un programa dado de la manera más eficiente posible. Las necesidades de la gestión de la integración se ajustan perfectamente 
a la descripción que Les Metcalfe hizo de la gestión pública que funciona "en un contexto pluralista en el que no se puede dar por sentado el consenso respecto a las metas, en el que la autoridad está dispersa, en el que el conflicto es legítimo y en el que, de todas formas, los diferentes integrantes son interde- pendientes y tienen intereses comunes aun cuando apenas pueda percibirse" (METCALFE, 1993: 185). La tarea fundamental -y muy difícil-consiste en formar las capacidades para enfrentar también la incertidumbre del entorno y los problemas imprevisibles.

\section{* Instituto Europeo de Administración Pública, Maastricht.}

' La comparación entre Europa y América Latina, a este respecto, ha sido abordada en forma prácrica en el marco del programa de Formación Unión Europea-Grupo de Río para la Integración Regional, ejecutado por el Instituto Europeo de Administración Pública (IEAP) a través del Centro de Formación para la Integración Regional (CEFIR) en Montevideo. El presente articulo se basa en el capírulo del autor, Co-Director de dicho Programa, en la publicación del CEFIR "Gerenciando la Integración: implicaciones de los Procesos de Integración Regional para las Administraciones Nacionales de los Paises Miembros del Grupo de Río", de próxima aparición.

${ }^{2}$ Pelkmans propone los siguientes ejemplos de procesos de integración preferencialista:

El preferencialismo sectorial parcial (por ejemplo: los acuerdos de complementariedad).

La integración sectorial:

- Preferencialista (por ejemplo: el convenio Automotriz entre EEUU y Canadá).

- La integración tanto positiva como negativa (por ejemplo: la Comunidad Europea del Carbón y del Acero).

Sistemas preferenciales no reciprocos:

- Parcial (por ejemplo: la mayoría de los acuerdos entre la CE y los países mediterráneos).

- Completa (por ejemplo: la Convención de Lomé)

Preferencialismo parcial recíproco (por ejemplo: ALADI),

Zona de libre comercio.

- Toda la industria (por ejemplo: EFTA).

- Todos los sectores (por ejemplo: CE-Israel, a pesar de las restricciones en agricultura).

Las distintas "etapas de la integración del mercado de productos" son:

- La unión arancelaria pura (comercio al interior de la unión libre de aranceles).

- La unión arancelaria-más (seguridad de despacho aduanero dentro de la unión).

- La pseudo unión aduanera (movimiento libre de los productos dentro de la unión, excepto en la esfera financiera ofiscal, o el libre acceso físico a los mercados).

- La unión aduanera pura (comercio al interior de la unión sin aduanas).

- Integración de los mercados de productos sin distorsiones (aproximación a

la unión aduanera como en la teoria: requiere uniformidad de las influencias públicas en condiciones competitivas).

${ }^{3}$ Un esfuerzo interesante por aplicar un marco teórico a una experiencia específica de integración en América Latina ha sido realizado por Augusto ANINAT (1992: 2-14) en su estudio para la CEPAL sobre la institucionalidad del Grupo
Andino.

4Algunas de éstas se encuentran entre las variables que afectan la estabilidad de los procesos de integración económica sugeridos por Pelkmans (1987). Corresponden también, en parte, a las tres "tareas variables clave" -alcance, incertidumbre e intensidad- identificadas como "indicadores de la complejidad" con respecto a las redes interorganizativas por ALter y HaGf. (1993). Varias pueden compararse también con las "variables críticas" para la eficacia de las instituciones internacionales sugeridas por Oran YouNG (1992): la eficacia varía directamente con la transparencia, la robustez de los mecanismos de elección social, lo estricto de las normas de transformación, la capacidad de los gobiernos y el nivel de interdependencia, pero está circunscripta por asimetrías profundas en la distribución del poder. Younc agrega el punto importante de que las "instituciones internacionales no pueden permanecer siendo eficaces durante mucho tiempo después de la erosión o el colapso de sus subestructuras intelectuales".

'Las implicaciones de la "dirección y la magnitud del ajuste de la política interna sustantiva" para las negociaciones intergubernamentales relativas a la asignación de funciones en los sistemas de discusión están bien analizadas por MORAVSCIK (1993).

${ }^{6}$ La "desestabilización" también puede afectar el dominio políico. En el ámbito institucional, en algunos países europeos, los parlamentos nacionales buscaron nuevas facultades o procedimientos para enfrentar los nuevos desafios planteados por la integración. La integración, a su vez, ha traido, en general, un aumento de las facultades ejecutivas a expensas de los órganos parlamentarios. Esto ha tenido el efecto de exacerbar algunos debates internos por lo que la ratificación del Tratado de Maastricht ofreció una apertura para el ajuste del balance interno de poderes. Esto resultó más claro en el caso de Francia, donde el Parlamento desempeñó un papel particularmente débil bajo la Constitución de la Quinta República y el "asunto europeo" constituía una buena ocasión para que el Parlamento levantara cabeza e hiciera saber al Ejecutivo que aceptaría menos fácilmente que en el pasado las limitaciones de poderes que le inflige la Constitución (Blumann, 1994: 397). Esto ha afectado también la relación entre las legislaturas y el público siendo más irónico quizás el caso del parlamento danés que tanto se ha vanagloriado de su control impecablemente democrático sobre el ejecutivo: si el Folketing fuera tan democrático, ¿cómo es posible que haya votado por Si el Tratado de Maastricht cuando el pueblo votó por No?

'Varios estudios han sido llevados a cabo en Europa con el objeto de comparar las capacidades nacionales, en general con un énfasis en la dimensión interministerial (Metcalfe y Zapico GoNì, 1987; Siedentopf y Ziller, 1988; Pappas, 1995). El Instituto Internacional de Ciencias Administrativas (IIAS) también realizó un programa de trabajo comparativo. 


\section{Bibliografia}

Catherine ALTER y Jerald HAGE (1993), Organizations Working Togetber(Newbury Park, London \& New Delhi: Sage).

Augusto AntNat del Solar (1992), "La institucionalidad en el Grupo Andino" (Santiago de Chile: CEPAL).

Michael M. AtKinson y William D. Coleman (1992), "Policy Networks, Policy Communities and the Problems of Govemance ${ }^{\circ}$, Governance 5:2 (April 1992): 34180 .

Edward BEST (1994), "Sistemas institucionales para la integración regional", Integración latinoamericana núm.. 202 (julio): 11-33.

C. Buumann (1994), "La ratification par la France du Traité de Maastricht", Revue du Marché commun et de l'Union européenne núm. 379 (juin): 393-406.

James A. CAPORASO (1974), The Structure and Function of European Integration (Pacific Palisades, CA: Goodyear).

CEPAL (Comisión Económica para América Latina y el Caribe) (1994a), El regionalismo abierto en América Latina y el Caribe (Santiago de Chile: Naciones Unidas).

CEPAL (1994b), El dinamismo reciente del comercio intrarregional de la Asociación Latinoamericana de Integración (ALADI) (Santiago de Chile, 23 de agosto).

M. HeRdeGen (1994), "Maastricht and the German Constitutional Count: Constitutional Restraints for an Ever Closer Union", Common Market Law Review, 31:2, 235-249.

Andrew HurRell (1995), "Regionalism in Theoretical Perspective" en Louise Fawcelt and Andrew Hurrell (eds.), Regionalism in World Politics. Regional organization and international order (Oxford: OUP): 37-73.

IDB (Inter-American Development Bank) (1995), Economic Integration in the Americas (Washington D.C., July).

Harold K. JACOBSON (1984), Networks of Interdependence. International Organizations and the Global Political System (New York: Alfred A. Knopf).

Edward D. MANSFELD (1993), "Effects of international politics on regionalism in intemational trade" en: Kym Anderson and Richard Blackhurst (eds.), Regional Integration and the Global Trading System (Harverster Wheatsheaf for GATT Secretariat, Geneva), pp. 199-217.

Les MetCAlfE (1993a), "Public management: from imitation to innovation", en: Kooiman (ed.), Modern Governance.

Les MetCalfe (1993b), "Gerencia de los procesos de integración: liderazgo, gestión y coordinación", Integración latinoamericana núms. 189-190 (mayojunio): 10-17.

Helen MILNER (1992), "International Theories of Cooperation among Nations. Surengths and Weaknesses", World Politics, 44:3 (April): 466-496.

Wim MOuE (1994), The Economics of European Integration, 2nd ed. (Aldershot: Dartmouth).

Andrew Moravcsik (1993), "Preferences and Power in the European Community: A Liberal Intergovernmentalist Approach", Joumal of Common Market Studies, 31:4 (Decemher): 473-524.
Gunnar P. Nielsson (1990), "The parallel national action process", en: A. J. R. Groom and Paul Taylor (eds.), Frameworks for International Co-operation (London: Pinter, 1990): 78-108.

Henri OBERDORfF (1995), "Des incidences de l'Union européenne et des Communautés sur le système administratif français", Revue du Droit Public et de la science politique en France et à l'étranger, 1-95 (Janvier-Février): $25-49$.

Spyros A. PAPpas (ed.) (1995), National Administrative Procedures for tbe Preparation and Implementation of Community Decisions (Maastricht: European Institute of Public Administration)

Jacques Pelkmans (1986), "The Institutional Economics of European Integra. tion", section III en Thomas Heller \& Jacques Pelkmans, "The Federal Economy: Law and Economic Integration and the Positive State - The U.S.A. and Europe Compared in an Economic Perspectiven, en Mauro Cappelletti, Monica Seccombe \& Joseph Weiler (eds.) Integration Througb Law. Vol.1 Book 1 (Berlin: de Gruyter).

Jacques Pelkmans (1987), "Alternative Methods of Economic Integration", en ASEAN at the Crossroads (Kuala Lumpur: ISIS): $421-436$.

Jacques Pelkmans (1993), “Comparando las integraciones económicas: prerrequisitos, opciones e implementaciones", Integración Latinoamericana núm.191 (julio): 3-17.

Jorge Pérez Otermin (1995), El Mercado Común del Sur. Desde Asunción a Ouro Preto (Montevideo: Fundación de Cultura Universitaria).

Robert D. Putnam (1988), "Diplomacy and Domestic Politics: The Logic of Twolevel Games", International Organization, vol. 42 (summer): 427-60.

Heinrich Siedentopf y Jacques Ziluer (eds.) (1988), Making European Policies Work. The Implementation of Community Legislation in the Member States Vol.1 Comparative syntheses (London: Sage).

Bengt Sundeulus (1978), Managing Transnationalism in Northern Europe (Boulder: Westriew).

Bengt Sundelius y Claes WIKLUnd (1979), "The Nordic Community: The Ugly Duckling of Regional Cooperation", Journal of Common Market Studies, XVIII:1 (September): 59-75.

Theo A. J. TOONEN (1992), "Europe of the Administrations: The Challenges of '92 (and Beyond)" Public Administration Review, 52:2 (March-April): 108-115.

Augusto DE LA TORRE y Margaret R. KFLLY (1992), Regional Trade Arrangements, (Washington, D.C.: International Monetary Fund).

WorLd BANK (1995), Global Economic Prospects and the Developing Countries (Washington D.C.).

Oran R. Young (1992), "The Effectiveness of International Institutions: Hard Cases and Critical Variables", en: James N. Rosenau \& Ernst-Otto Czempiel (eds.), Governance without government: order and change in world politics (Cambridge: CUP): 160-194.

Jacques ZILifr (1993), Administrations Comparées. les systèmes politico-administratifs de l'Europe des Douze (Paris: Montchrestien).

Jacques Zilufr (1994), “La culture des cadres nationaux et l'adaptation à l'Euro$\mathrm{pe}^{n}$, Revue française d'administration publique, núm. 70, pp. 241-251. 


\section{Muchos \\ de los directivos de la empresa española

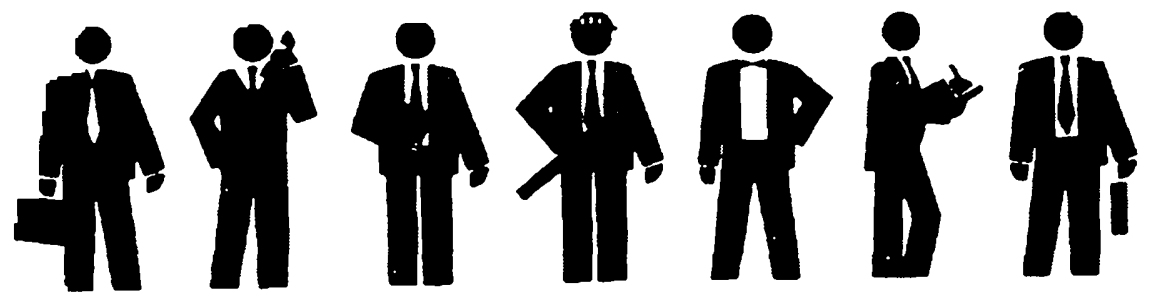 \\ se han formado en la EOI}

\section{Otros vienen para ponerse al día}

- Master en Gestión Industrial.

- Master en Gestión de Calidad.

- Master en Ingeniería y Gestión Medioambiental.

- Master en Dirección de Empresas.

- Master en Gestión de las Comunicaciones y Tecnologías de la Información.

- Master en Ingeniería Medioambiental. Gestión del Agua.

SEVILLA

Avda. n." 4. Isla de la Cartuja 41011) Sevilla

Tels.: (95) 4237567

Fax: (95) 4237682

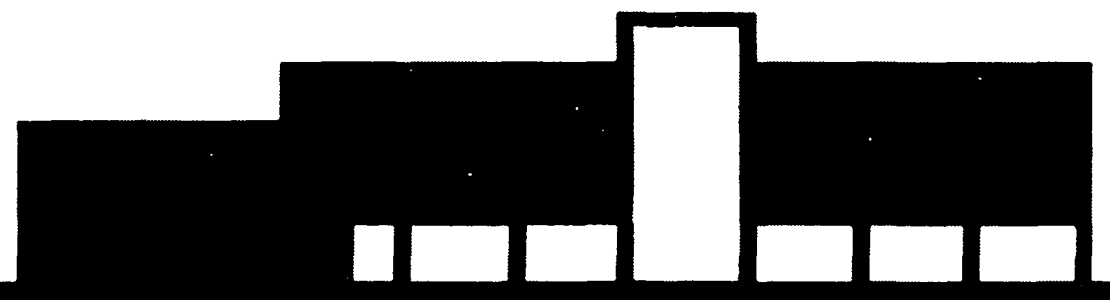

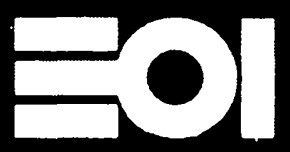

Ministerio de Industria y Energía
Escuela de

Organización

Industrial
Gregorio del Amo, 6

(Ciudad Universitaria). 28040 Madrid Tels.: (91) $3495656 / 00$ Fax: (91) 5542394 